\title{
Correction to: Prevalence of wormian bones in dried adult human skulls: an osteo-morphometric study in Nepal
}

\author{
Laju Maya Basnet $^{1}{ }^{1} \cdot$ Sunara Shrestha ${ }^{1} \cdot$ Subash Sapkota ${ }^{2}$
}

Published online: 23 August 2018

(c) Japanese Association of Anatomists 2018

\section{Correction to: Anatomical Science International https://doi.org/10.1007/s12565-018-0454-x}

In the original publication of the article, the affiliation of the coauthor Subash Sapkota and the formula of the cranial index were published incorrectly. The correct affiliation is provided in this correction.

The correct formula is given below:

Cranial index $(\mathrm{CI})=\frac{\text { Maximum cranial breadth }}{\text { Maximum cranial length }} \times 100$.

The original article can be found online at https://doi.org/10.1007/ s12565-018-0454-x.

Laju Maya Basnet

laju.basnet@gmail.com

1 Department of Anatomy, Nepal Medical College, Kathmandu University, Attarkhel, Gokarneswor Municipality-15, Post Box No. 13344, Kathmandu, Nepal

2 Department of Anatomy, Gandaki Medical College, Tribhuvan University, Pokhara, Nepal 